\title{
Defensiv oder offensiv? \\ Fallanalyse der sprachlichen Realisierungen von Argumentation anhand eines Sportinterviews
}

\section{Marie Krappmann}

The article focuses on the analysis of the linguistic means of argumentation in the dialogical form of argumentation. The theoretical basis for the analysis is the model developed by Wohlrapp (2008). A relatively prototypical example of a dialogical text type was chosen for the analysis of the argumentation-process: an interview between Holger Stanislawski, longtime trainer of the traditional football club St. Pauli, and a reporter. The article pursues two basic goals: 1) to verify the thesis by Wohlrapp that the argumentation processes are realised in simple repetitive argumentation moves; 2 ) to prove the fact that the argumentation strategies could partially be identified already on the level of linguistic expression.

Argumentative Operations - Linguistic Signalling of Argumentation - Particles - Reciprocal Argumentation

Der Beitrag konzentriert sich auf die Analyse sprachlicher Strategien im reziproken Argumentationsprozess. Dabei wird methodologisch auf den Ansatz von Harald Wohlrapp (2008) zurückgegriffen, der von sechs grundlegenden Argumentationszügen ausgeht. Für die Analyse der Argumentationsvorgänge wurde ein prototypisches Beispiel des medialen Dialogs ausgewählt: ein Interview mit dem langjährigen Trainer des traditionsreichen Fußballklubs St. Pauli, Holger Stanislawski. Mit der Analyse werden in erster Linie zwei Ziele verfolgt: 1) die These von Wohlrapp zu verifizieren, dass Argumentationen in einfachen, sich wiederholenden Schritten realisiert werden; 2) die Funktion der sprachlichen Realisierungen der einzelnen Argumentationsschritte zu überprüfen.

Argumentative Operationen - linguistische Signalisierung der Argumentation - Partikeln reziproke Argumentation

\section{1. "Neuentdeckung ${ }^{\mu}$ der Argumentation}

Argumentieren Sie niemals! Wer argumentiert, verliert. ${ }^{1}$

Mit diesen Worten werden potentielle Teilnehmer zu einem „unglaublichen Kommunikationsseminar“ eingeladen, für das sich „alle Menschen, die ihre Kommunikation im Berufs- und Privatleben konstruktiver, erfolgreicher und stressfreier gestalten möchten“, einschreiben sollten. In diesem Seminar soll man lernen, sich der Argumentation zu enthalten, um besser argumentieren zu können. Der einleitende Spruch mag natürlich eine werbewirksame Zuspitzung sein, trotzdem ist sein Aussagegehalt im gewissen Sinne symptomatisch für die zeitgenössischen Auseinandersetzungen mit dem Thema Argumentation. Die damit verbundenen Fragestellungen wurden in den letzten Jahrzehnten lebhaft diskutiert,

1 http://www.hd-beratung.de/bilder/PDFs/00510-Kommunikationsseminar.pdf (3. 4. 2013). 
nicht nur im Rahmen der wissenschaftlichen Argumentationsforschung, sondern auch in der wirtschaftlichen, politischen und medialen Sphäre.

Mit dem argumentativen Verfahren verbinden sich von jeher Fragestellungen, die noch heute aktuell sind. Es handelt sich um grundlegende Fragen wie etwa: Woran erkennt man argumentative Verfahren und wie definiert man sie? ${ }^{1}$ Wozu dient Argumentation?2 ${ }^{2}$ Mit welchen Mitteln kommt ein argumentatives Verfahren zustande? ${ }^{3}$ Es handelt sich zugleich um Fragen, die durchaus nicht erst mit der Entwicklung der modernen Argumentationsforschung aufgetaucht sind, sondern in den Forschungsbereichen der Rhetorik ${ }^{4}$ und Logik bereits lange im Raum stehen. Allerdings hat sich die moderne Argumentationsforschung über die Grenzen dieser beiden Disziplinen hinausentwickelt und ist zu einem breit angelegten interdisziplinären Forschungsfeld geworden. Van Eemeren, Grootendorst und Snoeck Henkemans definieren den Argumentationsvorgang im interdisziplinären Sinne als „verbal activity“, „social activity“ und „activity of reason“, 5 wobei diese Definition durchaus dem klassischen aristotelischen Verständnis der Argumentation entspricht.

Insbesondere seit den 70er Jahren erfreut sich die wissenschaftliche Forschung über Argumentationsstrukturen eines außergewöhnlichen Interesses, das sich auf mehreren Ebenen zeigt. Auf der einen Seite entstehen zahlreiche Arbeiten zu diversen Modellbildungen insbesondere im Bereich der Makrostrukturen der Argumentation. Diese Richtung haben in den 50er Jahren nachhaltig Stephen Toulmin (1958) mit seiner gebrauchstheoretischen Logikkritik und Chaim Perelman und OlbrechtsTyteca (1958) mit ihrem Konzept der „neuen Rhetorik“ geprägt. Seitdem sind zahlreiche argumentationstheoretische Modelle entstanden, ${ }^{6}$ die von jeweils sehr

1 Zur Abgrenzung von argumentativen Strukturen in der Alltagssprache wurden in den letzten Jahrzehnten zahlreiche Modelle entwickelt, die mit sehr unterschiedlichen methodologischen Ansätzen arbeiten. Zum detaillierten Übersicht der wegweisenden Ansätze in der modernen Argumentationsforschung siehe z. B. die Studie von Eemeren, Grootendorst und Snoeck Henkemans (1996: passim).

2 Insbesondere durch die Einbeziehung des pragmatischen Ansatzes in die Argumentationsforschung tauchten zahlreiche Fragen hinsichtlich der verschiedenen Funktionen der Argumentationsvorgänge auf. Die Definition der Argumentation etwa als ,das dialogische Überprüfen der mit den Forschungsthesen erhobenen Geltungsansprüche“ impliziert Fragen nach dem Handlungspotential der Argumentation, der Rollenverteilung usw. Zur Definition siehe etwa Wohlrapp (2008: 297).

3 In den letzten Jahrzehnten wurde die Rolle der sprachlichen Mittel in der Argumentation sehr kontrovers diskutiert. Einige Forscher schreiben der sprachlichen Signalisierung gar keine Relevanz zu: „Kurz: die argumentative Operation des Behauptens oder Thesen-Setzens dokumentiert sich nicht durch spezifische sprachliche Formen" (Wohlrapp 2008: 200). Andere vertreten wiederum den Standpunkt, die Argumentationsstrukturen seien aus der Sprachoberfläche deutlich ablesbar. Vgl. etwa die Studie von Atayan (2006: passim).

4 Zur geschichtlichen Entwicklung der Rhetorikforschung und ihrem Beitrag zur Argumentationsforschung vgl. z. B. Kienpointner (1992: 178-187).

5 Hier handelt es sich um die pragmadialektische Definition der Argumentation, so wie sie im Rahmen des sog. Amsterdamer Anasatzes formuliert wurde.

${ }_{6} \mathrm{Zu}$ den argumentationstheoretischen Klassifikationskriterien siehe Eemerens und Grootendorst (1992: 6ff). 
unterschiedlichen Definitionen des Begriffs „Argumentation“ ausgehen und sich mit sehr unterschiedlichen methodologischen Ansätzen dem Thema nähern. Dabei scheint die Unterscheidung zwischen Argumentationstheorien und empirischlinguistischen Argumentationsstudien sinnvoll, wobei die Grenze nicht immer klar definiert ist. Nach Bücker (2004: 2) bieten Argumentationstheorien ,aufgrund ihrer Distanz zu den sprachlichen Details argumentativer Interaktion und der Globalität ihrer Untersuchungsperspektive einen breiten Überblick über die möglichen Bereiche, in denen Argumentation thematisiert und zum Untersuchungsgegenstand gemacht werden kann." In Arbeiten, die (wie z. B. Toulmin 1958, Perelman 1958, Alexy 1978, Habermas 1987) in erster Linie auf Modellbildung ausgerichtet sind, wird daher eher deduktiv vorgegangen, mit Hypothesen ex ante. Andererseits entstehen zahlreiche auf Korpusdaten basierte Arbeiten (z. B. Kienpointner 1992, Selting und Couper-Kuhlen 2000, Atayan 2006), die eher sprachlich-kommunikativ ausgerichtet sind und dementsprechend geringere Ansprüche an die Modellbildung stellen. Kennzeichnend für diese Richtung sind etwa die Arbeiten aus dem Bereich der sog. interaktionalen Linguistik ${ }^{1}$, deren programmatische Grundlagen um das Jahr 2000 gelegt wurden. Es handelt sich um einen extrem empirisch ausgerichteten Ansatz, der größtenteils auf induktiven Untersuchungsmethoden basiert.

\section{Dialogizität - implizite Voraussetzung der Argumentation?}

Viele rezente Arbeiten, die sich mit Argumentationsstrukturen auseinandersetzen, versuchen allerdings, die sprachlich-kommunikative Perspektive mit modellbildenden Ansätzen sinnvoll zu kombinieren. Mit anderen Worten: Es werden aus verschiedenen Perspektiven Versuche unternommen, die theoretischen Modellbildungen mit der Alltagspraxis der Argumentation in Einklang zu bringen.

Stellvertretend für diese Vorgehensweise kann hier die Arbeit von Vahram Atayan (2006) erwähnt werden, der in seinen eher sprachzentriert ausgerichteten Analysen an die französischen Argumentationsforscher Oswald Ducrot (z. B. 1980; 1995; 2004) und Jean-Claude Anscombre (z. B. 1983; 1995; 2002) anknüpft. Diese haben die These des sog. "radikalen Argumentativismus“ entwickelt, welche die Analyse des argumentativen Verfahrens bereits auf der Ebene der konkreten sprachlichen Realisierung in der Form einer Äußerung ansetzt. Ducrot (2004:18f.) geht sogar von der Voraussetzung aus, dass alles Sprechen gleich Argumentieren sei. In seiner Theorie ist jede Äußerung argumentativ ausgerichtet, wobei sie die folgende Äußerung präselegiert. Atayan (2006) knüpft an dieses extrem sprachlich ausgerichtete Minimalmodell der Argumentation an, betont aber im stärkeren Maße die Handlungsfunktion der Sprache. In seiner Arbeitsdefinition des argumentativen Verfahrens wird die Funktion der sprachlichen Mittel und der pragmatischen Komponente hervorgehoben:

Die Weiterentwicklung dieser linguistischen Ausrichtung haben insbesondere die Aufsätze von Margret Setling und Elisabeth Couper-Kuhlen geprägt. Zu ihren Thesen siehe Selting und CouperKuhlen (2000: 76-95) und Selting und Couper-Kuhlen (2001: 257-287). 
„Eine minimale Argumentation besteht aus zwei (ggf. komplexen) kommunikativen Handlungen, die meistens auf der sprachlichen Oberfläche realisiert sind und zwischen denen eine vom Sender intendierte Stützungsrelation interpretativ angenommen wird“ (Atayan 2006: 41).

Obwohl Atayan seine Analysen hauptsächlich auf monologischen Argumentationssequenzen aufbaut, geht eine solche Definitionsabgrenzung offensichtlich von einer (zumindest implizit) dialogischen Struktur der Argumentation aus. Die implizite Dialogizität des Argumentationsverfahrens wird in den meisten Argumentationstheorien und -studien als ein grundlegendes Merkmal zur Identifizierung von Argumentationen angesehen. Atayan betont in diesem Kontext insbesondere die Funktion der Fremdsteuerung, die sehr oft als konstitutiver Prozess bei der Bildung argumentativer Makrostrukturen verstanden wird: „Als Erklärungsmechanismus [für die Fremdsteuerung der Makrostrukturen] wird hier häufig die Vermutung in den Mittelpunkt gestellt, argumentative Makrostrukturen seien das Resultat einer durch den Sender antizipierten NichtRatifikation in einer potentiellen Aushandlung" (Atayan 2006: 93). Mit anderen Worten: Die enttäuschten Erwartungen des Senders, die oft vorweggenommen werden, sind der zentrale Ausgangspunkt jeder Argumentation. Atayan macht allerdings darauf aufmerksam, dass viele Autoren monologische (nicht-reziproke) Texte für die Analyse der Argumentationsverfahren benutzen, in denen die sprachliche Signalisierung der Argumentationsstrategien deutlicher ausgeprägt sei. Den Grund dafür sieht er darin, dass bei dialogisch ausgerichteten Argumentationen der Wissensstand der Gesprächspartner die sprachliche Signalisierung zum Teil blockiert.

Der Zusammenhang zwischen der sprachlichen Signalisierung und dem Grad der Dialogizität leuchtet zwar im Kontext des radikalen Argumentativismus ein, ${ }^{1}$ allerdings stellen gerade dialogische Muster geradezu prototypische Beispiele für Argumentationsvorgänge dar. ${ }^{2}$ Überdies können manche Aspekte der Argumentation ausschließlich anhand von explizit dialogischen Texten untersucht werden. Der von Luhmann festgestellte Unterschied zwischen kognitiven und normativen Erwartungen ließe sich anhand von nicht-reziprok aufgebauten Texten nur schwer festlegen. Wenn wir mit Festinger (Festinger und Aronson 1960: 214-231) annehmen, dass argumentative Strukturen vordergründig die Funktion haben, Dissonanzen zwischen den Teilnehmern am Argumentationsverfahren auszugleichen, dann stellen eben die reziproken Texte eine ideale Untersuchungsbasis dar.

1 Das Ziel dieses Ansatzes ist es in erster Linie mit Hilfe von operationalen Verfahren nachzuweisen, dass sich der argumentative Wert einer Äußerung in der linguistischen Struktur selbst verbirgt. Zu einer konstruktiven Kritik dieses Ansatzes vgl. Iten (1999: 41-81).

2 Die Definitionen vieler Argumentationsforscher zielen genau in diese Richtung. So z. B. Wohlrapp, der Argumentieren als „das dialogische Überprüfen der mit den Forschungsthesen erhobenen Geltungsansprüche“ abgrenzt (Wohlrapp 2008: 297). 


\section{Sechs Anschlusszüge als strukturelle Basis von Argumentationsprozessen}

Einen effizienten Ansatz zur Analyse der reziprok aufgebauten Argumentationsstrukturen hat Harald Wohlrapp (2008) entwickelt. ${ }^{1}$ Auf der Grundlage des „minimalistischen Prinzips“2 in der Argumentationstheorie grenzt Wohlrapp (2008: 188) den theoretischen Apparat auf drei grundlegende Sprachhandlungsstrukturen ein, die im Prozess der Argumentation in der Alltagssprache ,immer auftreten, immer relevant sind und über die sich zu verständigen fundamental“ ist, nämlich die Grundoperationen Behaupten, Begründen und Kritisieren (Wohlrapp 2008: 192-214). Als vierte Struktur wird der „Rahmen“ definiert, der den kontextuellen Ausschnitt festlegt, innerhalb dessen der konkrete Argumentationsvorgang erfolgt. Es handelt sich im Grunde um eine räumliche Metapher, die sich grob mit anderen metaphorischen Ausdrücken für die Bezeichnung der Grenze des Bereichs, innerhalb dessen argumentiert wird, deckt, wie etwa Kontext, argument field usw. Wohlrapp schlägt allerdings hilfreiche Hinweise zur Eingrenzung eines dermaßen weit gefassten Begriffs vor, in dem er vier Strategien definiert, die in Argumentationsprozessen wirksam sind, nämlich Rahmenkritik, Rahmenhierarchisierung, Rahmenharmonisierung und Rahmensynthetisierung. ${ }^{3}$ Die Grundoperationen des Behauptens, Begründens und Kritisierens werden dabei in konkreten Diskussionen als Möglichkeiten von Zügen und Anschlusszügen betrachtet. Anhand dieser Überlegungen entwickelt Wohlrapp ein System von sechs echten Anschlusszügen, die in der Form von folgender Tabelle systematisiert werden können, in der die Zugmöglichkeiten der Opponenten- $(\mathrm{O})$ und Proponentenseite (P) zusammengefasst werden: ${ }^{4}$

1 Es handelt um einen relativ breit gefassten Ansatz, der einerseits im Unterschied zum logischanalytischen Zugang auch mit nicht-wahrheitsbedingten Kriterien arbeitet, andererseits die Einschränkung der Analyse z. B. auf sprachliche Vorgänge (vgl. die Thesen des radikalen Argumentativismus) meidet: „Eine argumentative Begründung setzt Freiheit voraus. Es scheint sich also bei der Theoretisierung des Argumentierens nicht allein um eine formale oder sprachstrukturelle Aufgabe zu handeln, sondern um ein Thema, das mit den großen philosophischen Themen zu tun hat" (Wohlrapp 2008: 212).

2 Die notwendige Folge des Minimalismus-Prinzips ist die Kritik an überflüssiger Theorie.

3 Zu Rahmenstrukturen vgl. Wohlrapp (2008: 237-296).

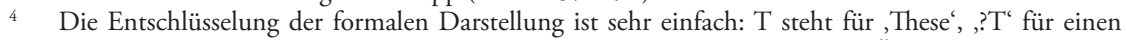
Zweifel daran, , $G$ 'steht für Begründungsschritt,,$G \rightarrow T$ ' für die annehmbare Übergangsmöglichkeit. ,A` bezeichnet ein von $T$ verschiedenes Argument oder eine abweichende These, , $A \rightarrow \neg T^{\prime}$ dann den Übergang von A zur Negation von T, , $B^{`}$ repräsentiert ein von T und A verschiedenes Argument (eine These), welches (welche) zwei weitere Übergangsmöglichkeiten aktiviert. Wohlrapp macht darauf aufmerksam, dass der Proponent im zweiten Zug durchaus mit ? $G^{\prime}$ anschließen könnte, was dann jedoch kein neuer Anschlusszug wäre. Für detaillierte Ausführungen siehe Wohlrapp (2008:300). 


\begin{tabular}{|l|l|l|}
\hline$P$ & $O$ & $P$ \\
\hline$T$ & $? T$ & $G(G \rightarrow T)$ \\
\hline & A $(A \rightarrow \neg T)$ & $? A$ \\
\hline & & $B(B \rightarrow \neg A)$ \\
\hline & & $G(A \rightarrow T)$ \\
\hline
\end{tabular}

Beim Erstellen dieses Systems geht Wohlrapp davon aus, dass dem Argumentierenden im Dialog an jeder Stelle im Grunde nur zwei Möglichkeiten des Anschlusszuges zur Verfügung stehen: Er kann das Argument des Dialogpartners übernehmen oder er kann es kritisieren. ${ }^{1}$ Dabei kann die Kritik zwei Formen annehmen: Das Argument kann entweder angezweifelt (?A) oder bestritten und durch ein anderes Argument ersetzt werden. Laut Wohlrapp kommen folgende Reaktionen auf einen Zweifel an A als Anschlusszüge in Frage: Das Argument kann zurückgezogen werden, oder es wird mit weiteren (koordinierten oder subordinierten) Begründungen weiter verteidigt. Auf das Bestreiten des Arguments (oder einer These) sind laut Wohlrapp insgesamt vier Anschlusszüge möglich: 1) Das Argument zurückziehen und das von der Oppositionsseite vorgebrachte neue Argument akzeptieren; 2) das neue Argument anzweifeln; 3) das neue Argument bestreiten und mit einem weiteren Gegenargument ersetzen; 4) das neue Argument durch Umdeutung integrieren.

Auf der Basis dieses durchaus einfachen Systems von Zugmöglichkeiten lassen sich die von Wohlrapp definierten Grundoperationen mühelos ergründen. Allerdings müssen auch verschiedene Ausweichstrategien in Betracht gezogen werden, die sich in diesem Schema nur schwer erfassen lassen. ${ }^{2}$ Das Problem besteht darin, dass auf der Ebene der sprachlichen Oberfläche gerade solche Ausweichmanöver intensiv signalisiert werden, die in Wohlrapps Ansatz eine eher sekundäre Rolle spielen. Dies wird auch explizit betont:

„Es geht mir gar nicht um eine bis ins Detail ausformulierte Theorie, die möglichst sogar bis in die sprachliche Oberfläche hinein reicht (etwa welche Wörter oder Formulierungen Argumentation indizieren, z. B. weil, also, deshalb, usw.), sondern es sollen nur gleichsam einige Schneisen angelegt werden, an denen sich das Prinzipielle zeigt [...]" (Wohlrapp 2008: 186).

\section{Medialer Dialog als spezifische Form der reziproken Argumentation}

Mit dem hier analysierten Argumentationsverfahren in einem Interview möchte ich indirekt an das Motto anknüpfen, mit dem der vorliegende Aufsatz eingeleitet wurde.

1 Der Argumentierende kann die Züge des Dialogpartners auch ignorieren, wodurch allerdings weitere Argumentation größtenteils blockiert wird.

2 Wohlrapp konzentriert sich hauptsächlich auf das kooperative Argumentieren, während Ausweichmanöver ein häufiges Mittel des strategischen Argumentierens darstellen. Zum Unterschied zwischen dem kooperativen und strategischen Argumentieren vgl. z. B. Völzing (1979: 13f). 
In reziproken Textgebilden kommen nämlich zahlreiche Argumentationsstrategien zum Einsatz, welche die grundlegende Funktion von Argumentationsprozessen bedeutend modifizieren. Laut Völzing soll die Funktion der Argumentation darin bestehen, „in Konflikten dazu zu dienen, gegenteilige Meinungen oder Ansichten herauszuarbeiten, per Kompromiss oder Konsens Lösungsmöglichkeiten anzubieten, oder auch Potential zur Verschärfung eines Konflikts bereitzuhalten “ (Völzing 1979: 12). Laut Völzing wird allerdings in zahlreichen, vor allem medialen Dialogen diese grundlegende Funktion nur scheinbar erfüllt. Dies hängt mit den Erwartungen zusammen, die an solche Dialoge gestellt werden. Völzing macht darauf aufmerksam, dass Texttypen wie Interview oder Diskussion in erster Linie „dazu dienen, prominenten Leuten brisante Informationen zu entlocken, um diese der Öffentlichkeit zugänglich zu machen" (Völzing 1979: 20). Nicht die Suche nach Lösungsmöglichkeiten sei in diesem Fall für die Entwicklung der reziproken Struktur ausschlaggebend, sondern die Intention, dem Gesprächspartner möglichst viele Informationen zu entlocken. Diese Strategie macht sich bereits auf der Ebene der sprachlichen Realisierung bemerkbar.

Um diese Annahme zu überprüfen, wurde für die folgende Analyse ein relativ prototypischer Vertreter der Textsorte „Interview“ ausgewählt. Der kontextuelle Rahmen ist folgender: Holger Stanislawski, der langjährige Trainer des Traditionsclubs FC St. Pauli, wechselt zu einem neuen Verein (Hoffenheim). Bei diesem Verein handelt es sich um einen neuen Club, der zwar Erfolge feiert, jedoch keine Tradition aufzuweisen hat und nur dank der finanziellen Unterstützung eines Mäzens überleben kann. Der Wechsel des Trainers ist daher keine Routineangelegenheit, sondern es handelt sich - metaphorisch ausgedrückt - um einen Frontenwechsel, der mit zahlreichen Emotionen seitens der Fans, der Mannschaften und des Trainers selbst verbunden ist. Daher bin ich der Meinung, dass sich dieses Interview der von Völzing angenommenen Handlungsstruktur in medialer Kommunikation entzieht und dass die Hauptintention tatsächlich ein Meinungsaustausch ist. Die folgende Analyse versucht, das von Wohlrapp vorgeschlagene Schema mit einer adäquaten Analyse sprachlicher Signalisierungen zu vereinen, wie sie etwa Atayan vorschlägt. Dabei wird der Akzent auf die verschieden Realisierungsmöglichkeiten von Ausweichmanövern gelegt.

\section{Die Fouls auf dem Feld der Argumentation - eine Fallanalyse ${ }^{1}$}

[Reporter - R 1]: Herr Stanislawski, Sie wechselten vor der Saison vom FC St. Pauli zur TSG aus Hoffenheim. Wäre es auch zu diesem Wechsel gekommen, wenn der Klassenerhalt mit den Hamburgern ein Selbstläufer wäre?

Der analysierte Text findet sich unter http://www.fussballjunkies.de/index. php? page $=$ Thread $\&$ threadID $=28793$ (5. 6. 2013). Im vorliegenden Artikel wurde nicht das ganze Interview, sondern lediglich der Teil analysiert, der interessantes Material hinsichtlich des offensiven und defensiven Argumentationsverhaltens bietet. 
Bereits aus der ersten Frage geht deutlich hervor, dass der Reporter eine offensive Argumentationsstruktur aufbaut, indem er eine herausfordernde These ${ }^{1}$ formuliert: Dem Gesprächspartner wird unterstellt, dass er in erster Linie aus Berechnung handelt. Es handelt sich offenbar um eine im Grunde rein provokative Fragestellung, denn mit der Antwort „Nein“ würde sich der Befragte von vorne herein disqualifizieren. Der umgangssprachliche Ausdruck „Selbstläufer“ erweckt den Eindruck ungezwungener Gesprächsatmosphäre, in der sich die Mitteilsamkeit des Gesprächspartners steigern soll. Der eher legere Ton gehört außerdem durchaus zu den Kennzeichen der Textart „Sportinterview“. Aus der Perspektive der Argumentationsstruktur stellt sich die Frage, ob die konkrete Wahl des Lexems vielleicht ein Mittel der Fremdsteuerung darstellt. ${ }^{2,3}$ Die erste Frage ist also eine bewusste Herausforderung zum defensiven Sprachverhalten, auf die der Befragte fast gezwungen ist einzugehen. Die Partikel ${ }^{4}$ auch, die hier am ehesten mit selbst dann paraphrasierbar wäre, spielt hier auf der kommunikativ-pragmatischen Ebene eine interessante Rolle. Wenn man den Deletionstest durchführt und die beiden Sätze, mit und ohne auch, miteinander vergleicht, kann ein unauffälliger Unterschied festgestellt werden: Durch den Fragesatz mit auch wird die im folgenden Nebensatz ausgedrückte Bedingung fokussiert. Dadurch wird der Zweifel des Fragenden an der erfragten Tatsache/ Tätigkeit zum Ausdruck gebracht, weswegen man eine Steigerung der Defensive beim Gefragten erwarten kann. ${ }^{5}$

Holger Stanislawski [S 1]: Meine Entscheidung von Pauli nach Hoffenheim zu wechseln war vollkommen unabhängig vom sportlichen Erfolg oder Misserfolg mit Pauli. Es ging mir vielmehr darum, mich nach über zehn Jahren bei einem Club beruflich zu verändern, etwas Neues kennenzulernen.

S. geht - wie erwartet - in die Defensive über, indem er die Äußerung vom R. reformuliert und den Zusammenhang zwischen den Spielleistungen von St. Pauli und seinem Wechsel negiert. Die defensive Reaktion wird durch das hier als

1 Auf der sprachlichen Ebene wird allerdings die argumentative Stärke der Äußerung durch den konditionalen Nebensatz und den Konjunktiv im Hauptsatz abgeschwächt.

2 Eine Reaktion wie etwa: „Nein, wenn der Klassenerhalt ein Selbstläufer gewesen wäre, wäre es zu diesem Wechsel nicht gekommen "wäre sicherlich sehr überraschend. Das umgangssprachliche und daher expressivere Lexem stärkt meines Erachtens die Erwartung einer bejahenden Antwort.

3 Zur Funktion der deutschen Abtönungspartikel im Kontrast zum Tschechischen vgl. Rinas (2006: 331-339). Zum Lexem aber in der Funktion der Partikel vgl. Rombouts (1982: 65-67).

4 Der Begriff „Partikel“ wird im vorliegenden Aufsatz als Bezeichnung für eine Gruppe unflektierbarer Wörter verwendet, nicht als Bezeichnung der spezifischen Gruppe „Abtönungspartikeln“.

5 Im Satz Wärest du zu der Party auch gegangen, wenn Paul nicht da gewesen wäre? wird mit Hilfe von auch angedeutet, dass die/der Fragende eher der Meinung ist, die gefragte Person hätte in diesem Fall nicht an der Party teilgenommen. Burkhardt (1982) definiert für auch in der Funktion einer Abtönungspartikel in Entscheidungsfragen eine beinahe umgekehrte pragmatische Wirkung: In der Frage des Weihnachtsmannes Wart ihr auch artig? zweifelt dieser nur scheinbar am normadäquaten Verhalten, präsupponiert eher mit Hilfe von auch die Erwartbarkeit der Normadäquatheit und macht dadurch eine positive Antwort wahrscheinlicher. Vgl. Burkhardt (1982: 101). 
Gradpartikel gebrauchte Wort vollkommen hervorgehoben, das jedoch in diesem Kontext eher Unsicherheit markiert. Bereits Wilhelm Scheuerle (1971: 280) hat darauf aufmerksam gemacht, dass der Gebrauch von bestimmten Lexemen und Wendungen, die aus linguistischer Sicht meistens als Modalwörter (zweifellos, selbstverständlich), Gradpartikeln (vollkommen, total, etwas) oder Partikeln (durchaus) klassifiziert werden, sehr häufig auf das Verdecken der Unsicherheit oder des Nicht-Wissens hindeutet. ${ }^{1}$ Ein weiterer Abgrenzungsversuch von der Motivation „Berechnung“, die Stanislawski von dem Reporter implizit unterstellt wird, spiegelt sich in der expliziten Nennung der beiden Zustände: „Erfolg oder Misserfolg“ wider. Auch dieses Vorgehen signalisiert auf der sprachlichen Ebene ein gesteigertes Defensivverhalten.

Als der „wirkliche“ Grund für den Wechsel wird die zu lange andauernde Tätigkeit bei St. Pauli angeführt und die daraus resultierende Sehnsucht nach neuen Herausforderungen. S verhält sich hier deutlich nach dem Schema A (A $\rightarrow$ $\neg T)$. Durch das adversative Adverb vielmehr, das den im zweiten Satz angeführten Grund mit dem negierten Grund im ersten Satz verbindet, wird zugleich die Relevanz des zweiten Grundes betont. Diese Akzentuierung scheint - zumindest auf der kommunikativ-pragmatischen Ebene - ein ähnliches Indiz zu sein wie die Partikel vollkommen: Die verstärkt defensive Position weist auf Unsicherheit hin. Die konkrete sprachliche Realisierung des komplexen Gegenarguments, die sehr abstrakt ausfällt - „sich beruflich zu verändern“ und „etwas Neues kennen lernen“-, signalisiert ein stark defensives Sprachverhalten, das bereits an ein Ausweichmanöver grenzt.

$\boldsymbol{R}$ 2: Pauli ist ein Verein, dem man eine sehr ausgeprägte Fangemeinschaft und eine enorme Tradition bescheinigt. Der TGS wird oftmals nachgesagt, dass sie eine "gekaufte" Tradition besitzt und ein Eventpublikum zu Gast hat. Wie stehen Sie dazu und wie sieht ein Vergleich beider Fankulturen aus?

In der zweiten Frage wird eine neue offensive These vorgetragen, die allerdings implizit eine thematische Fortsetzung der ersten darstellt. ${ }^{2}$ Indem das „richtige“ Publikum von St. Pauli in Kontrast zum „unechten“ Hoffenheimer Publikum gestellt wird, bildet der Reporter eine Dichotomie, die sich implizit auch auf die Beurteilung der Entscheidung überträgt, die der Befragte getroffen hat. Im Grunde versteckt sich hinter einer so formulierten These der Argumentationszug A.. ${ }^{3}$ Der Kontrast zwischen den beiden Fangemeinden wird durch expressive Attribute hervorgehoben. Es stehen auf einer Seite „ausgeprägte“ Fangemeinschaft und „enorme“ Tradition, auf der anderen Seite „gekaufte“ Tradition und „Eventpublikum “. Es wird also eine

Diese Meinung vertritt auch Völzing (1979: 224).

Deswegen ist der erste Teil gar nicht als Frage, sondern als eine Feststellung formuliert.

Das Argument [zu lange Betätigungszeit] wird in Frage gestellt und implizit durch B [höheres Verdienst] ersetzt. Dadurch wird ein neuer Argumentationszug formuliert: $\mathrm{B}(\mathrm{B} \rightarrow \neg \mathrm{A})$. 
Dichotomie von Stabilität und Treue einerseits und Instabilität und Bestechlichkeit andererseits impliziert. Dabei distanziert sich der Reporter zugleich von dieser Dichotomie mittels der Passivkonstruktion „der TSG wird nachgesagt“. Dadurch legitimiert er die Aussage über einen Teil der Dichotomie durch die Meinung einer abstrakten Mehrheit. Nach dieser Feststellung folgt die eigentliche Frage, in der Stanislawski aufgefordert wird, die Feststellung zu kommentieren.

S2: Man kann die beiden Clubs nur schwer miteinander vergleichen. In Hoffenheim stehen wir am Anfang, die Fankultur befindet sich im Aufbau und muss sich dementsprechend noch entwickeln. Aber wir sind auf einem guten Weg.

Der Befragte reagiert mit einem zu erwartenden Ausweichmanöver, in dem er die Möglichkeit des Vergleichs in Frage stellt. Mit dem allgemeinen Subjekt man wird die Allgemeingültigkeit der Aussage suggeriert. Um die Ausweichstrategie zu tarnen, wird ein kausaler Zusammenhang zwischen dem Alter des jeweiligen Clubs und der Fankultur hergestellt. Der Befragte ignoriert die implizite Struktur B (B $\rightarrow \neg \mathrm{A}$ ) und stellt eine neue These $\mathrm{T}$ auf. Mit den metaphorischen Konzepten des gerade eingeschlagenen Weges und des kaum angefangenen Gebäudes ${ }^{1}$ wird eher eine indirekte Parallele zu St. Pauli gezogen. In diesen Konzepten spielt die zeitliche Dimension eine ausschlaggebende Rolle: Die beiden Metaphern evozieren das Ziel, zu dem ein wohl geplantes Vorgehen führen soll. Sie implizieren lediglich eine graduelle, zeitlich bedingte Differenz zwischen den Fangemeinden und negieren den vom Reporter angedeuteten Wesensunterschied. Die Partikel noch in der syntaktischen Funktion eines Temporaladverbs unterstreicht dieses metaphorische Konzept. Es verweist zwar darauf, dass das Ziel bisher nicht erreicht ist, impliziert aber zugleich, dass zu dessen Erreichung nur wenig fehlt (vgl. Métrich/Faucher 2009: 621f.).

Eine auffallende, sprachlich ausgedrückte Veränderung der Sprecherposition wird von dem Wechsel in die erste Person Plural signalisiert. Dadurch soll offenbar die Zugehörigkeit des Befragten zum Hoffenheimer Club betont werden, wodurch er sich zugleich auf eine unauffällige Art und Weise von der ehemaligen Mannschaft (St Pauli) distanziert. Ob die Zugehörigkeit wirklich vom Sprecher empfunden wird oder nicht, spielt dabei keine Rolle. Insbesondere in medial ausgetragenen Debatten handelt es sich häufig eher um ein strategisches Manöver. ${ }^{2}$

$\mathrm{Zu}$ diesen metaphorischen Konzepten vgl. Lakoff und Johnson (2003: 73-78).

„Das Personalpronomen „wir" kann also einen Zustand der Identifikation, des Einklangs von P (Produzenten) und R (Rezipienten) oder von P und anderen, für die er spricht, gegenüber oder auch mit R bzw. der von $\mathrm{R}$ vertretenen Gruppe bezeichnen, es kann aber auch dazu eingesetzt werden, einen Zustand des Einklangs da zu suggerieren, wo er nicht gegeben ist“ (Völzing 1979: 230). 
R 3: Dietmar Hopp glich erst im vergangenen Geschäftsjahr einen enormen Schuldsaldo des Vereins aus. Viele fragen sich, ob die TSG überhaupt selbstständig überlebensfähig wäre oder noch stark vom Mäzen abhängig ist. Wie sehen Sie diese Thematik?

Auch in der folgenden Frage, die mit einer Konklusion eingeleitet ist, wird seitens des Reporters ein Kontrast zwischen dem vom Geld des Mäzens (Dietmar Hopp) abhängigen Hoffenheimer Club und den anderen, „überlebensfähigen“ Clubs der Bundesliga hergestellt. Die Berechtigung der darauf folgenden Frage nach der finanziellen Überlebensfähigkeit der Mannschaft wird einerseits wieder durch den Verweis auf das Interesse der abstrakten Menge gestützt (,viele fragen sich“), andererseits durch den Hinweis auf das Ausmaß der Schulden mit Hilfe des expressiven Attributs enorm. Im Grunde wird mit dieser Konklusion die innerhalb der zweiten Frage aufgestellte Dichotomie zwischen den beiden Clubs weiter verfolgt. Formal ausgedrückt: Es wird der in der zweiten Frage formulierte Grund B (Kontrast zwischen FC St. Pauli und Hoffenheim) wieder aufgenommen, wodurch $\operatorname{der}$ Zug B $(\mathrm{B} \rightarrow \neg \mathrm{A})$ in reformulierter Form unterschwellig aktiviert wird.

In der anschließenden Frage werden von dem Reporter zwei Möglichkeiten angeboten: Hoffenheim kann im Moment selbständig finanziell überleben / Hoffenheim kann dies nicht. Auch hier wird die Antwort antizipiert: Es wir offenbar nicht erwartet, dass von dem Befragten eine der angebotenen Möglichkeiten direkt gewählt wird. Die kontrastive Fragestellung stellt eine stark ausgeprägte offensive Strategie dar. Dabei wird die Haltung des Reporters durch den Gebrauch der sprachlichen Mittel angedeutet: Die abtönungsähnliche Partikel überhaupt $t^{l}$ hat in der Entscheidungsfrage auf der kommunikativ-pragmatischen Ebene hauptsächlich die Funktion, die Proposition grundsätzlich in Frage zu stellen (vgl. Weydt 1983: 21). König (1983: 161f.) spricht von „negative[n] Polaritätskontexte[n]“, die mit distributionell eingeschränkten Elementen wie je(mals) vereinbar sind. Der Reporter, der sich hinter der abstrakten Menge „versteckt“, zweifelt also an der Möglichkeit der finanziellen Unabhängigkeit des Hoffenheimer Clubs.

Noch in der Funktion einer Fokuspartikel ${ }^{2}$ drückt hier zugleich eine zeitliche Relation aus: Der Zustand der starken Abhängigkeit dauert an, seine allmähliche Abschwächung (oder gar sein Ende) ist aber abzusehen. Der Gebrauch von noch in der zweiten alternativen Funktion eröffnet dem Befragten die Möglichkeit, wieder auf die zeitliche Dimension hinzuweisen, die er bereits in der Antwort auf die zweite Frage signalisierte.

1 Bereits König (1983) hat festgestellt, dass überhaupt in keine der Subklassen wie Konjunktionen, Fokuspartikeln oder Abtönungspartikeln passt. Er hat sich daher für die Kategorisierung „Adverb“ entschieden (König 1983: 160), während Weydt das Wort in der gleichen Publikation als „abtönungsähnliche Partikel“ klassifiziert (Weydt 1983: 21). Ziffonun/Hoffmann/Strecker (1997: 59) definieren das Wort später als Konnektivpartikel.

2 Zur Übersicht vgl. Métrich/Faucher (2009: 623). 
$S$ 3: Wir arbeiten hart daran, den Club finanziell auf eigene Füße zu stellen. Auch hier brauchen wir sicherlich noch etwas Zeit, ich wüsste aber nicht, warum wir das nicht schaffen sollen. Unser Gehaltsniveau haben wir fast soweit gesenkt wie nötig, hinzu kommt die positive Transferbilanz im vergangenen Jahr.

Das defensive Verhalten des Befragten weist die gleiche Struktur auf wie bei der Antwort auf die zweite Frage. Die These $\mathrm{T}$ wird in einer reformulierten Form wiederholt: Der wesentliche Unterschied wird negiert, der graduelle, zeitlich bedingte Unterschied zugegeben. Dabei wird wieder die einschließende Form der 1. Person Plural verwendet, die weiterhin konsequent benutzt wird.

Im ersten Argumentationsschritt geht der Befragte dankbar auf das Argument der zeitlichen Dimension ein. Zuerst wird der gerade stattfindende Prozess betont, der zum ersehnten Ziel führen soll. Sprachlich wird dies durch eine Metapher ausgedrückt, die sich konzeptuell mit der Weg- und Gebäudemetapher deckt. Etwas, was noch nicht auf eigenen Füßen steht, muss diesen Zustand erst erreichen. Als Mittel wird zunächst abstrakt „die harte Arbeit“ angeführt. Anschließend wird das geringe Alter des Clubs hervorgehoben, genauso wie bei der zweiten Frage. Die Parallelität wird dabei durch die Wendung „auch hier“ betont. Es wird also implizit die zweite vom Reporter aufgeworfene Möglichkeit bejaht (der Club ist vom Mäzen abhängig), aber zugleich der Übergang zu der ersten Möglichkeit (finanzielle Unabhängigkeit) impliziert. Das Modalwort ${ }^{1}$ sicherlich indiziert dabei wieder eher argumentative Unsicherheit des Befragten als eine feste Überzeugung. Diese Annahme wird auch durch die konjunktivische Form der nächsten Aussage unterstützt. $^{2}$

Um dem relativ abstrakten Defensivverhalten eine Basis zu verleihen, werden zwei Beispiele als Argumente für die Konklusion [Erzielen der Unabhängigkeit in absehbarer Zeit] angeführt. Nun wird „die harte Arbeit“ konkretisiert - das Senken des Gehaltsniveaus und die positive Transferbilanz. ${ }^{3}$

R 4: Im letzten Jahr wurde ein erneuter Versuch, die "50+1-Regel" abzuschaffen abgeblockt. Sehen Sie dies als Niederlage im internationalen Vergleich oder zeigt Deutschland gerade durch seine Jugendarbeit, dass es auch ohne Teilhaber-Millionen aus den Emiraten geht?

$1 \quad$ Die Terminologie bei der Bezeichnung von Partikeln wie vielleicht, zweifellos, sicherlich u. a., die die subjektive Einstellung des Sprechers zum Ausdruck bringen, schwankt erheblich. Während sie etwa Latour (1997: 112) oder Ballweg (2006: 547-554) als „Modalpartikeln“ bezeichnen, bestimmen sie Helbig und Buscha als „Modalwörter“ (Helbig/Buscha 1994: 500-512), deren Aufgabe es ist, die Proposition in Bezug auf ihren Wahrheitswert zu graduieren oder sie emotional zu bewerten.

2 Die Aussage ,ich wüßte aber nicht, warum wir das nicht schaffen sollen“ soll - in der Terminologie von Toulmin - das Postulat der Ausnahmebedingung negieren ( $\rightarrow$ Es gibt keine Bedingung, die für das Nichtgelingen des Prozesses gültig wäre.) Vgl. Toulmin (1996: 88-98).

3 Die Intensitätspartikel fast (unser Gehaltsniveau haben wir $\mathrm{f}$ a $\mathrm{s}$ t so weit gesenkt wie nötig) unterstreicht die zeitliche Dimension - es evoziert nämlich ein weiteres Senken des Gehaltsniveaus. 
Die nächste Frage stellt einen weiteren Angriff dar, der immer noch von der aufgestellten Dichotomie zwischen den beiden Clubs ausgeht. Die Fragestellung wird mit einer kontextuell gebundenen Feststellung eingeleitet: der Versuch, die 50+1-Regel abzuschaffen, wurde abgeblockt. ${ }^{1}$

Die darauf folgende Frage wird sehr indirekt formuliert, indem weder auf die Situation in FC St. Pauli noch auf die Lage in Hoffenheim näher eingegangen wird. Der Reporter bietet $S$ wieder zwei Alternativen als Konsequenzen der nicht zugelassenen 50+1 Regel an, von denen die eine deutlich negativ markiert wird. 1) Die erste Alternative ist die Niederlage im internationalen Vergleich. Diese Alternative impliziert folgenden Argumentationsübergang: Deutschland hat den Versuch der Abschaffung der 50+1-Regel abgeblockt (Argument ${ }^{2}$ ), die anderen Staaten werden im Vorteil sein (Konklusion). 2) Die zweite Möglichkeit stellt von der argumentativen Perspektive einen Grund für das weitere Beharren auf der 50+1-Regel dar. Als Argument wird die gute Jugendarbeit erwähnt. Die Argumentationslinie könnte man folgendermaßen paraphrasieren: Deutschland kann aus eigenem Nachwuchs gute Spieler rekrutieren (Argument I), daher werden keine Gelder „von außen“ benötigt (Konklusion I) und die 50+1-Regel wird (trotzdem) keinen negativen Einfluss auf die Stellung des deutschen Fußballs im internationalen Vergleich haben (subordinierte Konklusion II).

Die Fokuspartikel gerade spielt bei der Auslegung des Argumentationsvorganges eine besonders wichtige Rolle. Nach der traditionellen illokutionären Interpretation dieser Partikel nimmt der Sprecher an, dass der Hörer der Meinung ist, die Jugendarbeit sei nicht so wichtig (vgl. Bartsch 1979: 371). Der Reporter scheint diese zweite Alternative implizit zu befürworten (und zu verteidigen) und grenzt sich zugleich von der ersten Alternative (Niederlage im internationalen Vergleich) ab.

Die negative Markierung kann auch auf der lexikalischen Ebene festgestellt werden: Die Formulierung „Teilhaber-Millionen aus den Emiraten" wurde offenbar absichtlich gewählt, um einen möglichst entfernten kulturellen Rahmen zu evozieren, der auf Grund des islamischen Kontextes zusätzlich als bedrohlich empfunden wird. Die adverbiale Partikel auch funktioniert als eine weitere emotionale Markierung: In diesem Kontext signalisiert sie deutlich die Identifizierung des Reporters mit der

1 Die 50+1-Regel ist ein in den Statuten der Deutschen Fußball-Liga festgelegter Paragraph, der eine Übernahme der Stimmen-Mehrheit durch Investoren bei Fußball-Vereinen untersagt. Die Mehrheit des Kapitals dagegen darf den Anlegern gehören. Durch diese normative Regelung soll verhindert werden, dass die Profimannschaften von Vereinen unter die vollständige Kontrolle von Großunternehmen oder anderen Kapitalgebern geraten, wie es etwa aus England bekannt ist. Das Ziel ist, den Vorrang der sportlichen Interessen der Vereine vor den wirtschaftlichen Interessen der Investoren zu wahren.

2 Mit dem Begriff „Argument“ wird in den Studien über Argumentationstheorie oft Verschiedenartiges bezeichnet. Im vorliegenden Aufsatz ist damit konsequent der Teil des Argumentationsvorgangs gemeint, der den Übergang zur Konklusion ermöglicht. 
zweiten Alternative. Im Bezug auf das Modell von Wohlrapp wird also T1 und T2 aufgestellt, wobei T2 als die „richtige“ These markiert wird.

$S$ 4: Über den Sinn der $50+1$-Regel sollen sich andere Gedanken machen. Die Jugendarbeit in Deutschland aber ist hervorragend, was allein durch den Jugendstil in der Nationalmannschaft bewiesen wird. Wir verfolgen in Hoffenheim ein ganz ähnliches Konzept und treten in der Bundesliga mit einem der jüngsten Teams überhaupt an.

Der Befragte reagiert mit einem explizit zugegebenen Ausweichmanöver: Er disqualifiziert sich selbst, indem er auf seine mangelnde Kompetenz hinweist und die Verantwortlichkeit weitergibt. Dadurch wird die eigentlich gestellte Frage (und ein weiterer potentieller Argumentationszug) abgeblockt. Im Anschluss daran geht das explizite Ausweichmanöver in ein implizites über. Der Befragte ignoriert nämlich den Übergang vom Argument gute Jugendarbeit zur Konklusion I es werden keine Gelder von außen benötigt. Er reagiert lediglich auf das erste Argument, dessen Gültigkeit er bestätigt. Dieses Argument - gute Jugendarbeit wird für den Übergang zu einer neuen Konklusion verwendet, nämlich Jugendstil der Nationalmannschaft. Dabei wird auf die Konklusionen, die aus der Frage des Reporters hervorgehen, gar nicht eingegangen. Stattdessen wird ein neuer Argumentationsübergang aufgestellt: Die Jugendarbeit in Deutschland ist gut $\rightarrow$ Es gibt viele junge Spieler in der Nationalmannschaft. Auf diese Weise wird bewusst der Ausgangspunkt für die Ziehung einer Parallele zwischen der in Deutschland sehr geschätzten Nationalmannschaft und der Hoffenheimer Mannschaft geschaffen, indem auf das gemeinsame Merkmal [hoher Anteil an jungen Spielern] hingewiesen wird. Die Aufmerksamkeit der Rezipienten wird auf diese Weise von dem Übergang vom Argument zur Konklusion (Wenn gute Jugendarbeit $\rightarrow$ dann keine Beteiligung von außen) auf das bloße Merkmal [Jugend] gelenkt, das von dem Befragten positiv markiert wird. Dass es sich hierbei von Seiten des Befragten um taktisches Vorgehen handelt, wird auf der sprachlichen Ebene relativ deutlich signalisiert. Auf der lexikalischen Ebene wird das sehr abstrakte Lexem "Jugendstil“ benutzt, um konkrete Aussage zu vermeiden. Der argumentative Übergang vom Argument gute Jugendarbeit in Hoffenheim zur Konklusion Jugendstil der Hoffenheimer Mannschaft ist ungenügend gestützt. Obwohl in Hoffenheim intensiv mit der Jugend gearbeitet wird, befindet sich im aktuellen Kader der ersten Mannschaft kein Spieler, der aus der Hoffenheimer Juniormannschaft übernommen worden wäre. ${ }^{1}$ Dadurch kann auch die beabsichtigte Parallele zwischen Nationalmannschaft und Hoffenheim in Frage gestellt werden. Deswegen das bewusst abstrakt gewählte Lexem.

Die Partikel aber in dem Satz „Die Jugendarbeit in Deutschland aber ist hervorragend" soll hauptsächlich das auffällige Ausweichmanöver verdecken. In dieser Position indiziert die adversative Partikel eine Gegenüberstellung bzw.

$\overline{1}$ Die TGS Hoffenheim ist im Gegenteil häufig Vorwürfen ausgesetzt, sie würde anderen Vereinen talentierte junge Spieler abwerben. Der Verein wehrt sich meistens mit dem (zutreffenden) Argument, dies sei eine in der Bundesliga gängige Praxis. 
Einschränkung. Genau dies will hier S. argumentativ erzielen, nämlich sich vom Thema "Gelder von außen“ distanzieren und die Aufmerksamkeit auf das Merkmal [Jugend] lenken. Die Fokuspartikel allein erfüllt eine ähnliche Funktion. ${ }^{1}$ Im vorliegenden Kontext hat sie keine restriktive, sondern eine evaluative Funktion: Alternativen als Argumente für die Proposition werden nicht ausgeschlossen. Der Satz ließe sich paraphrasieren als ,Auch andere Kriterien als Jugendstil beweisen die hervorragende Jugendarbeit.' Jugendstil wurde als ein exemplarisches Beispiel ausgewählt. Dadurch wehrt S. im Vornhinein die eventuellen Angriffe gegen den nicht genügend gestützten argumentativen Übergang ab.

Nachdem die Aufmerksamkeit der Rezipienten mit Hilfe der erwähnten sprachlichen Mittel von der falschen Konklusion abgelenkt worden ist, kann schließlich die Parallele zwischen „dem Konzept" der Nationalmannschaft und Hoffenheim gezogen werden. Das Streben nach dieser Parallele seitens des Befragten hat durchaus seine Begründung, die im außersprachlichen Kontext zu suchen ist: $\mathrm{Zu}$ der Zeit, als das Interview aufgenommen wurde, feierte die Nationalmannschaft bedeutende Erfolge. Durch das Ziehen dieser Parallele sollte also auf den zukünftigen Erfolg der Hoffenheimer Mannschaft hingewiesen werden. Dabei wird dem Rezipienten die Annahme suggeriert, dass eine möglichst junge Mannschaft ein Garant für den Erfolg sei. Der Befragte hat also durch eine Reihe von Ausweichmanövern auf argumentativer und sprachlicher Ebene erreicht, dass die ursprünglich gestellte Frage in den Hintergrund tritt.

Mit diesem Ausweichmanöver auf der Ebene der Argumentationszüge erzielt $S$. bei R. endgültig die Resignation auf die Offensive: Der Reporter verzichtet in der nächsten Frage auf weitere offensive Argumentationsschritte, wobei der Übergang zur kooperativen Argumentationsart explizit ausgedrückt wird: „Kommen wir zum Sportlichen und zur TSG.“

\section{Schlussfolgerungen}

Die durchgeführte Analyse hat zu folgenden Schlussfolgerungen geführt, die einerseits das Analysemodell von Wohlrapp zu bestätigen scheinen, andererseits die maßgebliche Rolle von sprachlichen Indikatoren in den Argumentationsprozessen bestätigen:

I) Es hat sich gezeigt, dass die argumentative Reaktion des Gesprächspartners von der konkreten Ausformulierung der Fragestellungen gelenkt wurde, was insbesondere in medialen Texten häufig der Fall zu sein scheint.

II) Das absurd anmutende Motto in der Einleitung des vorliegenden Beitrags hat sich zum Teil bewährt: Dadurch, dass der Befragte bewusst irreführende

1 Zur Funktion von Fokuspartikeln im Deutschen vgl. etwa Thurmair (1989: 17f). Detaillierte Überlegungen zur Funktion des Lexems allein vgl. Brauße (2000). 
argumentative Übergänge konstruiert oder verschiedene implizite und explizite Ausweichmanöver vornimmt, verhindert er im Weiteren die für ihn unangenehme offensive Argumentation. Daher ist es nahezu unmöglich zu bestimmen, wer die Diskussion "verloren“ oder "gewonnen“ hat, da die Argumentationsschritte außerhalb der erwarteten Bewertungsmaßstäbe erfolgen.

III) Die These von Wohlrapp, dass die Argumentationsvorgänge in einfachen, sich wiederholenden Argumentationszügen realisiert werden, hat sich bestätigt. Bei mehreren, scheinbar neuen Argumentationszügen der beiden Gesprächspartner ließ sich feststellen, dass es sich um strukturell identische Reformulierungen der bereits vorgetragenen Argumente und Konklusionen handelt.

IV) Die Argumentationsstrategien konnten zum Teil bereits auf der Ebene der sprachlichen Äußerung identifiziert werden, indem eine Analyse bestimmter relevanter sprachlicher Mittel vorgenommen wurde. Es hat sich also gezeigt, dass auch in den einzelnen Sequenzen der reziprok aufgebauten Texte die sprachliche Signalisierung der Argumentationsverfahren deutlich ausgeprägt ist.

\section{Literaturverzeichnis}

Alexy, Robert (1978): Theorie der juristischen Argumentation. Die Theorie des rationalen Diskurses als Theorie der juristischen Begründung. Frankfurt a.M., Suhrkamp.

Anscombre, Jean-Claude / Ducrot, Oswald (1983): L'argumentation dans la langue. Bruxelles, Madraga.

Anscombre, Jean-Claude (Hg.) (1995): Théorie des topoï. Paris: Éditions Kimé.

Anscombre, Jean-Claude (2002): Mais/pourtant dans la contre-argumentation directe: raisonnement, généricité et lexique. In: $\operatorname{Linx} 46$. S. 115-131.

Atayan, Vahram (2006): Makrostrukturen der Argumentation im Deutschen, Französischen und Italienischen. Frankfurt am Main, Peter Lang.

Ballweg, Joachim (2006): Modalpartikel. In: Hoffmann, Ludger (Hg.): Handbuch der deutschen Wortarten. Berlin, Walter de Gruyter.

Bartsch, Renate (1979): Wahrheitsbedingungen und andere Gebrauchsbedingungen. In: Weydt, Harald (Hg.): Die Partikeln der deutschen Sprache. Berlin / New York, de Gruyter.

Brauße, Ursula (2000): Die Partikel allein. Klassifizierungs- und Bedeutungsprobleme. In: Linguistik online 6, 2/00. http://www.linguistik-online.de/2_00/brausse.html (2. 3. 2013).

Bücker, Jörg (2004): Argumentationstheorie und interaktionale Linguistik. SASI Heft 1. S. 2. http//noam.uni-muenster.de/SASI (12. 4. 2013).

Burkhardt, Armin (1982): Abtönungspartikeln als Mittel des Vollzugs präsuppositionaler Akte. Zu Dittmanns und Rombouts' Untersuchungen über die Abtönungsfunktion von auch, denn und doch. In: Zeitschrift für germanistische Linguistik 10, S. 85-112.

Ducrot, Oswald (1980) : Les échelles argumentatives. Paris, Les Editions de Minuit.

Ducrot, Oswald (1995): Les Modificateurs déréalisants. In: Journal of Pragmatics 24. S. $145-165$. 
Ducrot, Oswald (2004): Argumentation rhétorique et argumentation linguistique. In: Doury, Marianne / Moirand, Sophie (Hg.): L'argumentation aujourd'hui. Positions théoriques en confrontation. Paris, Presses Sorbonne Nouvelle.

Eemeren, Frans van / Grootendorst, Rob / Snoeck Henkemans, Francisca (1996): Fundamentals of argumentation theory. A handbook of historical backgrounds and contemporary developments. Mahwah, N. J., Lawrence Erlbaum.

Eemerens, Frans H. / Grootendorst, Rob (1992): Argumentation, Communication and Fallacies. A pragma-dialectal Perspective. Hillsdale, N.J., Lawrence Erlbaum.

Festinger, Leon / Aronson, Elliot (1960): Arousal and Reduction of Dissonance in Social Contexts. In: Cartwright, Dorwin / Zander, Alvin (Hg.): Group Dynamics. Evanston, IL: Row, Peterson.

Habermas, Jürgen (1987): Theorie des kommunikativen Handelns, Band 1: Handlungsrationalität und gesellschaftliche Rationalisierung. Frankfurt a. M., Suhrkamp.

Helbig, Gerhard / Buscha, Joachim (1994): Deutsche Grammatik. Ein Handbuch für den Ausländerunterricht. Leipzig, Langenscheidt.

Iten, Corinne (1999): The Relevance of Argumentation theory. In: UCL Working Papers in Linguistics 11, S. 41-81. http://www.phon.ucl.ac.uk/home/PUB/WPL/99papers/iten. $\operatorname{pdf}(23.4 .2013)$.

Kienpointner, Manfred (1992): Alltagslogik. Strukturund Funktion von Argumentationsmustern. Stuttgart / Bad Cannstatt, frommann-holzboog.

Lakoff, George / Johnson, Mark (2003): Metaphors We Live By. London, University of Chicago Press.

Latour, Bernd (1997): Deutsche Grammatik in Stichwörtern. Stuttgart, Klett Edition Deutsch.

Métrich, René/ Faucher, Eugéne (2009): Wörterbuch deutscher Partikeln. Unter Berücksichtigung ihrer französischen Äquivalente. Berlin / New York, de Gruyter.

Perelman, Chaim / Olbrechts-Tyteca, Lucie (1958): Traité de l'argumentation - La Nouvelle rhétorique. Bruxelles, Éditions de l'Université de Bruxelles.

Rinas, Karsten (2006): Abtönungspartikeln, dass Mittelfeld und die Wackernagelposition. In: Brücken. Germanistisches Jahrbuch Tschechien - Slowakei 2006. Bonn: DAAD, S. 331-339.

Rombouts, Jos (1989): Kann man Abtönungspartikel paraphrasieren? In: Zeitschrift für germanistische Linguistik 10. S. 63-84.

Scheuerle, Wilhelm (1971): Juristische Evidenzen. In: Zeitschrift für Zivilprozess 84/3. S. 241-297.

Selting, Margret / Couper-Kuhlen, Elizabeth (2000): Argumente für die Entwicklung einer „interaktionelen Linguistik“. In: Gesprächsforschung - On-line-Zeitschrift zur verbalen Interaktion 1. S. 76-95. http://www.gespraechsforschung-online.de/heft2000/ga-selting. pdf (13. 3. 2013).

Selting, Margret / Couper-Kuhlen, Elizabeth (2001): Forschungsprogramm „Interaktionale Linguistik“. In: Linguistische Berichte 187. S. 257-287.

Thurmair, Maria (1989): Modalpartikeln und ihre Kombinationen. Tübingen, Max Niemeyer Verlag.

Toulmin, Stephen (1958): The Uses of Argument. Cambridge, Cambridge University Press. Deutsche Übersetzung: Toulmin, Stephen (1996): Der Gebrauch von Argumenten. Weinheim: Athenäum Verlag.

Völzing, Paul-Ludwig (1979): Begründen, Erklären, Argumentieren. Modelle und Materialien zu einer Theorie der Metakommunikation. Heidelberg, Quelle \& Meyer. 
Wohlrapp, Harald (2008): Der Begriff des Arguments. Über die Beziehungen zwischen Wissen, Forschen, Glauben, Subjektivität und Vernunft. Würzburg, Königshausen \& Neumann.

Weydt, Hentschel (1983): Partikeln und Intonation. Tübingen, Niemeyer.

Zifonun Gisela / Hoffmann, Ludger / Strecker Bruno et al. (1997): Grammatik der deutschen Sprache. Berlin / New York, de Gruyter.

\section{Internetquellen}

http://www.hd-beratung.de/bilder/PDFs/00510-Kommunikationsseminar.pdf (3. 4. 2013). http://www.fussballjunkies.de/index.php?page=Thread $\&$ threadID=28793 (5. 6. 2013)

Mgr. Marie Krappmann, PhD.

Katedra germanistiky FF UP

Kř́ž́zovského 10

CZ-772 00Olomouc

marie.krappmann@upol.cz 